\title{
Client preferences and acceptability for medical abortion and MVA as early pregnancy termination method in Northwest Ethiopia
}

\author{
Mulatu A Woldetsadik', Tegbar Y Sendekie ${ }^{2}$, Mary T White ${ }^{3}$ and Desalegn T Zegeye ${ }^{4^{*}}$
}

\begin{abstract}
Background: Increasing access to safe abortion services is the most effective way of preventing the burden of unsafe abortion, which is achieved by increasing safe choices for pregnancy termination. Medical abortion for termination of early abortion is said to safe, effective, and acceptable to women in several countries. In Ethiopia, however, medical methods have, until recently, never been used. For this reason it is important to assess women's preferences and the acceptability of medical abortion and manual vacuum aspiration (MVA) in the early first trimester pregnancy termination and factors affecting acceptability of medical and MVA abortion services.

Methods: A prospective study was conducted in two hospitals and two clinics from March 2009 to November 2009. The study population consisted of 414 subjects over the age of 18 with intrauterine pregnancies of up to 63 days' estimated gestation. Of these 251 subjects received mifepristone and misoprostol and 159 subjects received MVA. Questionnaires regarding expectations and experiences were administered before the abortion and at the 2week follow-up visit.

Results: The study groups were similar with respect to age, marital status, educational status, religion and ethnicity. Their mean age was about 23, majority in both group completed secondary education and about half were married. Place of residence and duration of pregnancy were associated with method choice. Subjects undergoing medical abortions reported significantly greater satisfaction than those undergoing surgical abortions ( $91.2 \%$ vs 82.4\%; $P$ < .001). Of those women who had medical abortion, $(83.3 \%)$ would choose the method again if needed, and $(77.4 \%)$ of those who had MVA would also choose the method again. Ninety four percent of women who had medical abortion and $86.8 \%$ of those who had MVA would recommend the method to their friends.
\end{abstract}

Conclusions: Women receiving medical abortion were more satisfied with their method and more likely to choose the same method again than were subjects undergoing surgical abortion. We conclude that medical abortion can be used widely as an alternative method for early pregnancy termination.

\section{Background}

Unsafe abortion is a public health problem. Globally, 20 million unsafe abortions take place each year and account for $13 \%$ of all maternal deaths [1]. In Ethiopia $32 \%$ of all maternal death is due to unsafe abortion [2]. In addition, unsafe abortion accounts for nearly $60 \%$ of all gynecologic admissions and almost $30 \%$ of all obstetric and gynecologic admissions in Ethiopia [2,3]. In Ethiopia, as of May 2005, the law on abortion was

\footnotetext{
* Correspondence: zdesalegn@gmail.com

${ }^{4}$ Department of Epidemiology, College of Medicine and Health Sciences,

University of Gondar, Gondar, P.O.Box-196, Ethiopia

Full list of author information is available at the end of the article
}

revised in Article 551 of the Penal Code, to include four legal grounds in which pregnancy can be terminated: rape or incest, lethal congenital malformation, physical and mental deficiency to bring up the child, and if continuation of pregnancy endangers the life of the mother or the child or the health of the mother [4]. According to the law, no consent from a spouse, partner or parent is required to obtain a legal abortion and no requirements exist for legal reporting or documenting rape or incest as a prerequisite for obtaining a legal abortion [4].

Based on the revised law, the Federal Ministry of Health of Ethiopia developed technical and procedural guidelines for safe abortion services [2]. According to

\section{C) Biomed Central}


the national guidelines on safe abortion services, the Family Health Department of the Ministry of Health of Ethiopia, and partners like Ipas are collaborating to expand access to comprehensive abortion services. The introduction of low cost pre-packaged preparation of mifepristone and misoprostol, Medabon ${ }^{\circledR}$ is underway. The preparation consists of $200 \mathrm{mg}$ mifepristone (one tablet), to be taken on day 1 , and $0.8 \mathrm{mg}$ misoprostol (four tablets) to be taken on day 2 or day 3 .

When properly administered, abortion is one of the safest procedures in contemporary medical practice. So, increasing access to safe abortion services is the most effective way of preventing the burden of unsafe abortion $[5,6]$.

For a new technology to be a viable option in a given health care system, even when services are free, people need to want to use it and to demand it. For this reason, understanding how potential patients perceive and value a new technology is important $[7,8]$. In the case of medical abortion, however, patient acceptability is key to the success of the method [6,7,9-11]. If a procedure is acceptable, woman would choose it again if they needed to terminate another pregnancy and would recommend it to their friends [12]. Indeed, the combination of mifepristone-misoprostol for early abortion is established as safe, effective, and acceptable to women in several developing countries. In Ethiopia, however there was no study conducted on the choice and preference of medical method of abortion and MVA.

For this reason, it is timely and important to assess women's preferences for, and the acceptability of, medical abortion and MVA in the early first trimester pregnancy termination and factors respectively affecting the acceptability of medical and MVA abortion services.

\section{Methods}

A prospective study was conducted from March 2009 to November 2009. The study was conducted in 4 health institutions located in North Gondar, East and West Gojjam, where both medical abortion and MVA were provided. Four health institutions, namely, Gondar University Hospital, DebreMarkos Hospital, Marystopes clinics of DebreMarkos and Bahirdar were included. Other Government Hospitals and health institutions were not selected because the service was not fully started during the data collection period. Sample size of 414 was reached for the study, assuming greater than 10,000 eligible women will seek to terminate pregnancy in the selected institutions during the study period, $5 \%$ of level of significance, $5 \%$ margin of error, and that $57.2 \%$ of clients prefer medical method of terminating pregnancy [8]. Expecting a non-response rate of 10\% (since abortion is sensitive issue) and maintaining high statistical power, the final required sample size became
414 women. Women with gestational age $\leq 63$ days from their last menstrual period, with abdominal ultrasound or by physical examination $\leq 9$ weeks, who sought abortion and fulfilled the criteria of the Revised Abortion Law of Ethiopia [4], and who came during the study period, were eligible for the study. Those who had contraindications to medical or surgical abortion were excluded from the study. Those who were not able to provide information due to language barriers or cognitive incapacitation were also excluded.

All clients who requested termination of early pregnancy were given the same oral information about the two methods without a recommendation of one method over the other.

The medical abortion protocol consisted of $200 \mathrm{mg}$ mifepristone at the first visit, followed by $800-\mu$ g vaginal misoprostol 48 hours later, and a follow up visit scheduled after 14 days. Backup surgical abortion was provided to women experiencing medical method failure and complications. Women in the surgical group of the study received the standard procedure of vacuum aspiration with local anaesthesia. Follow up visit for a surgical client was scheduled 14 days after the procedure. All procedures were done by trained providers.

Eight data collectors who were nurses in the respective facilities but not on duty received training for one day collect the data. A pretested and structured interview questionnaire was used as the instrument of data collection. Data collected at the first visit were on demographic characteristics and reasons for choice of the procedure. A follow up questionnaire assessed the actual abortion experience. Data were collected on overall satisfaction, future choice of an abortion method, acceptability (acceptability of medical and MVA abortion in this study is re-use/recommendation to a friend), side effects, and place of possible pregnancy expulsion.

Quality of data was assured by using a structured questionnaire, which was translated to Amharic and pre-tested before the actual survey, and by training data collectors prior to the survey. In addition, regular supervision and checking filled data for completeness, clarity and accuracy was made every day by the investigators.

Data were coded, entered and cleaned, using SPSS version 13 statistical package. Some variables were recorded into different variables then analysis was done. Frequencies, percentages, cross tabulations and Chisquare tests were used for socio demographic and obstetrics characteristics, method choice and reasons for selecting the method, acceptability, abortion experience and satisfaction. In order to test for the associations of the outcome variables against the independent variable multiple logistic regressions was applied accordingly the p-value, confidence interval and odds ratio were computed and interpreted. 
Ethical clearance for the study was obtained from the Institutional Review Board of the University of Gondar. The study subjects were informed about the purpose of the research, the benefit and the risk of participation, the privacy and confidentiality of the study and their right to withdraw at any time from the study and this had no effect on their treatment. Verbal consent was obtained from each subject.

\section{Results}

A total of 414 women were included in the study. Four incomplete questionnaires were excluded from analysis.

The study groups were similar with respect to age, marital status, educational status, religion and ethnicity. The mean age of women who opted for the medical method was 23.1 years (range: $15-40$ years) and that of MVA was 23.7 years (range: 15-43 years) respectively. However, more women from urban areas (64\%) chose medical abortion as compared to the rural residents (51\%). (Table 1).

The majority of study participants had no child previously $(67.3 \%$ of women who chose medical abortion and $59.7 \%$ of women who chose MVA). About $85 \%$ of both groups reported no history of previous abortion. The duration of pregnancy was less than 7 weeks in 174 (69.3\%) women who chose medical abortion and 89 (56.0\%) of those who chose MVA (Table 2)

Table 1 Socio-demographic characteristics of the respondents by method choice in four selected health institutions, Northwest Ethiopia, November, 2009

\begin{tabular}{ccl}
\hline Variable & Medical & MVA \\
\hline $\begin{array}{c}\text { Age (mean) } \\
\text { Place of residence* }\end{array}$ & $23 \pm 4.4$ & $23.7 \pm 4.8$ \\
Urban & $204(81.3)$ & $115(72.3)$ \\
Rural & $47(18.7)$ & $44(27.7)$ \\
Marital status & & \\
Single & $122(48.6)$ & $71(44.7)$ \\
Married & $119(47.4)$ & $84(52.8)$ \\
Other & $10(4.0)$ & $4(2.5)$ \\
Educational level & & \\
Illiterate & $37(14.7)$ & $35(22.0)$ \\
Primary & $40(15.9)$ & $31(19.5)$ \\
Secondary and above & $174(69.3)$ & $93(58.5)$ \\
Religion & & \\
Orthodox & $236(94.0)$ & $144(90.6)$ \\
Muslim & $10(4.0)$ & $12(7.5)$ \\
Other & $5(2.0)$ & $3(1.9)$ \\
Ethnicity & & \\
Amhara & $245(97.6)$ & $153(96.2)$ \\
Oromo & $4(1.6)$ & $3(1.9)$ \\
Other & $2(.8)$ & $3(1.9)$ \\
\hline
\end{tabular}

Table 2 Obstetrics characteristics of the respondents by method choice in four selected health institutions, Northwest Ethiopia, November, 2009

\begin{tabular}{ccc}
\hline Variable & Medical & MVA \\
\hline Nulliparae & $67.3 \%$ & $59.7 \%$ \\
Number of children (mean $\pm \mathrm{sd}$ ) & $2 \pm 1.3$ & $2.2 \pm 1.7$ \\
Ever had abortion & $14.7 \%$ & $15.1 \%$ \\
Duration of current pregnancy* (mean $\pm \mathrm{sd}$ ) & $46 \pm 8.9$ & $49 \pm 8.9$ \\
\hline $\mathrm{p}<\mathbf{0 . 0 2}$ & &
\end{tabular}

\section{Method choice}

Overall 251(61.2\%) women chose medical abortion and 159(38.8\%) opted for MVA. Among women who selected the medical method, $120(47.8 \%)$ did so primarily to avoid pain and $19(7.6 \%)$ chose to avoid surgery or anesthesia. In contrast, women choosing surgical abortion did so mainly because it entailed fewer visits 102 (64.2\%) and was believed to be safer $80(31.9 \%)$ than the medical method of pregnancy termination (Table 3).

Binary logistic regression was conducted to examine factors associated with method choice. Those women who were living in urban area were 1.64 times more likely to choose medical abortion than MVA and women with duration of pregnancy less than 49 days were 1.77 times more likely to choose medical abortion than MVA (not shown on table).

\section{Acceptability and abortion experience}

There were 13(5.2\%) failures of medical abortion and 7 (4.4\%) MVA failures. Of the side effects, abdominal cramp, nausea and vomiting were common in both methods but more common in medical than MVA. With regard to place of abortion $182(76.5 \%)$ of medical abortions took place at home compared with $3(2.0 \%)$ of MVA. The majority of women had less than seven days of bleeding in both methods of abortion (Table 4).

Table 3 Choice of method and the reasons for selecting their method of respondents in four selected health institutions, Northwest Ethiopia, November, 2009

\begin{tabular}{cc}
\hline Variable & Percentage \\
\hline Reason to choose medical & 47.8 \\
To avoid pain & 31.9 \\
Safer & 7.6 \\
To avoid surgery or anesthesia & 6.8 \\
Fear of side effect & 6 \\
Others & \\
Reason to choose MVA & 64.2 \\
Fewer visit & 20.8 \\
Simpler and faster & 6.3 \\
To avoid side effect & 7.5 \\
More effective & 1.3 \\
Others &
\end{tabular}


Table 4 Abortion experience of respondents by method in four selected health institutions, Northwest Ethiopia, November 2009

\begin{tabular}{lcl}
\hline Variable & Medical & MVA \\
\hline Pregnancy Expelled & $94.4 \%$ & $92.5 \%$ \\
Place of abortion & & \\
$\quad$ Health facility & $50(21 \%)$ & $144(98 \%)$ \\
$\quad$ Home & $182(76.5 \%)$ & $3(2 \%)$ \\
$\quad$ Could not identify location & $4(2.1 \%)$ & \\
Other & $1(0.4 \%)$ & \\
Duration of bleeding*(mean $\mathbf{n}$ sd) & $6.7 \pm \mathbf{5 . 2}$ & $3.6 \pm \mathbf{3 . 7}$ \\
Side effects & & \\
Abdominal cramp* & $85.7 \%$ & $66 \%$ \\
Nausea* & $56.2 \%$ & $27.7 \%$ \\
Vomiting* & $25.1 \%$ & $15.1 \%$ \\
Diarrhea & $3.6 \%$ & $0.6 \%$ \\
Fever & $11.6 \%$ & $6.3 \%$ \\
Headache & $13.1 \%$ & $15.7 \%$ \\
\hline
\end{tabular}

${ }^{*} P<0.01$

The majority of women were satisfied with their abortion experience, but those women who had medical abortion were more satisfied than MVA (91.2\% vs. $82 \%$ ). Most women who received abortion services said they would choose the method again should the need arise, $83.3 \%$ medical abortion and $77.4 \%$ MVA respectively. Nearly all (94\%) medical abortion clients and $86.8 \%$ of MVA abortion clients would recommend their method to their friends. However, there was no significant difference between medical abortion and MVA users with regard to future choice of the method and recommendation of the method to friends (Table 5).

\section{Discussion}

This study of 410 women represents the first report for early first trimester pregnancy termination undertaken in Ethiopia. In our study, 251(61.2\%) women chose medical method. This is consistent with another study in Vietnam where $66.2 \%$ of subjects preferred medical

Table 5 Distribution of abortion patients, by measure of satisfaction and acceptability with their method, according to method in four selected health institutions, Northwest Ethiopia, November, 2009

\begin{tabular}{ccc}
\hline Variable & Medical & MVA \\
\hline Satisfaction & & \\
Satisfied with the method* & $91.2 \%$ & $82.4 \%$ \\
Reason for dissatisfaction & & \\
Heavy bleeding & $7(31.8 \%)$ & $5(17.9 \%)$ \\
Method failure & $10(45.5 \%)$ & $7(25 \%)$ \\
Pain & $5(22.7 \%)$ & $16(57.1 \%)$ \\
Will choose the method again & $83.3 \%$ & $77.4 \%$ \\
Will recommend the method * & $94 \%$ & $86 \%$ \\
\hline$*_{p}<0.05$ & &
\end{tabular}

abortion and 33.8\% opted for MVA [13]. Similarly, in the United Kingdom, 57\% preferred medical and 42.8\% opted for MVA [8]. Most women living in urban areas and those women in early pregnancy opted for medical abortion over MVA in our study. The reason could be those women who were living in rural areas want to avoid repeated visit to the health institution. This finding supported by similar study done in India where medical abortion was more commonly opted by women belonging to urban areas and in early pregnancy $(86.6 \%$ vs $46.6 \%$ ) [14].

Regarding the location of the abortion, $76.5 \%$ of medical abortion took place at home. The reason could be women were not kept at health institution for the first 4 to 6 hours after taking mifepristone by which $90 \%$ of abortion occurs. In addition to this, in our study the total duration from administration of misoprostol to possible expulsion of pregnancy was not collected. This finding is different from similar study done in Vietnam were $72 \%$ of medical abortion took place in the clinic [13].

In our study, $91.2 \%$ of women using the medical method and $82.4 \%$ of women using MVA were satisfied. Similarly, $83.3 \%$ of those who used the medical method and $77.4 \%$ of those who used MVA would opt for the same method of treatment if they were ever to have another termination of pregnancy. However, there was no statistically significant difference among the two groups. The reason why the medical method was not selected by all in our study could be side effects like abdominal pain, nausea and vomiting were more common in medical than MVA client. A study done in Vietnam showed women who had medical abortion were more likely to select the same method again than were those who selected MVA(96\%vs.52\%) [13].

With regard to recommendation of the method to their friends, those women who chose medical abortion would recommend their method slightly more often compared with those who choose MVA (94\% vs86.8\%). This may be due to those women who had abdominal pain were less likely to recommend MVA to their friends, but this had no influence on recommendation of medical method to friends. Our results are similar to studies done in Finland, Denmark and UK $[8,11,15]$.

In our study side effects like abdominal pain and nausea affected acceptability of both methods, hence counselling about women's expectations and appropriate pain control should be provided.

In this study some of the findings had wide confidence interval; this could be due to small number of cases in some of the cells.

\section{Conclusions}

This study showed that medical method for termination of early pregnancy was chosen more compared with 
MVA and the choice of medical abortion was associated with duration of pregnancy and place of residence. Similarly, both medical abortion and MVA were accepted and most women were satisfied by both methods. Acceptability may be affected by side effects of abortion methods.

\section{Acknowledgements}

We would like to thank our data collectors for their invaluable effort without them this study would not have come to be completed. Our deep gratitude also goes to our study subjects who were volunteered and took their time to give us all the relevant information for the study.

\section{Author details}

'Department of Gynaecology and Obstetrics, College of Medicine and Health Sciences, University of Gondar, Gondar, P.O.Box-196, Ethiopia. ${ }^{2}$ JHPIEGO Ethiopia, Addis Ababa, Ethiopia. ${ }^{3}$ Department of Community Health, Wright State University, Dayton, USA. ${ }^{4}$ Department of Epidemiology, College of Medicine and Health Sciences, University of Gondar, Gondar, P.O. Box-196, Ethiopia.

\section{Authors' contributions}

MAW wrote the proposal, participated in data collection, analyzed the data and drafted the paper. DTZ, TYS and MTW approved the proposal with some revisions, participated in data analysis and revised subsequent drafts of the paper. All authors read and approved the final manuscript.

\section{Competing interests}

The authors declare that they have no competing interests.

Received: 12 February 2011 Accepted: 3 June 2011

Published: 3 June 2011

\section{References}

1. WHO: Unsafe abortion: global and regional estimates of incidence of unsafe abortion and associated mortality in 2003. Geneva: World Health Organisation; 2007

2. FMOH: Technical and Procedural Guidelines for Safe Abortion Services in Ethiopia. Addis Ababa: Federal Ministry of Health Ethiopia; 2006.

3. Singh S: Hospital admissions resulting from unsafe abortion: estimates from 13 developing countries. Lancet 2006, 368(9550):1887-1892.

4. FDRE: The Criminal Code of the Federal Democratic Republic of Ethiopia. Crimes against life unborn: Abortion; 2004:Section II:219-221.

5. WHO: Unsafe Abortion: Global and Regional Estimates of the Incidence of Unsafe Abortion and Associated Mortality. Geneva: World Health Organisation; 2007

6. Berer M: Medical abortion: issues of choice and acceptability. Reproductive Health Matters 2005, 13(26):25-34.

7. Jensen JT, Harvey SM, Beckman LJ: Acceptability of suction curettage and mifepristone abortion in the United States: a prospective comparison study. American Journal of Obstetrics and Gynecology 2000, 182(6):1292-1299.

8. Child TJ, Thomas J, Rees M, MacKenzie IZ: A comparative study of surgical and medical procedures: 932 pregnancy terminations up to 63 days gestation. Human Reproduction 2001, 16(1):67-71.

9. Loeber OE: Motivation and satisfaction with early medical vs. surgical abortion in the Netherlands. Reproductive Health Matters 2010, 18(35):145-153.

10. Dao B, Blum J, Thieba B, Raghavan S, Ouedraego M, Lankoande J, Winikoff B: Is misoprostol a safe, effective and acceptable alternative to manual vacuum aspiration for postabortion care? Results from a randomised trial in Burkina Faso, West Africa. Bjog 2007, 114(11):1368-1375

11. Rørbye C, Nørgaard M, Nilas L: Medical versus surgical abortion: comparing satisfaction and potential confounders in a partly randomized study. Human Reproduction 2005, 20(3):834-838.

12. Winikoff B, Sivin I, Coyaji KJ, Cabezas E, Xiao B, Gu S, Du MK, Krishna UR, Eschen A, Ellertson C: Safety, efficacy, and acceptability of medical abortion in China, Cuba, and India: a comparative trial of mifepristonemisoprostol versus surgical abortion. American Journal of Obstetrics and Gynecology 1997, 176(2):431-437.

13. Ngoc N, Winikoff B, Clark S, Ellertson C, Am K, Hieu D, Elul B: Safety, Efficacy and Acceptability of Mifepristone-Misoprostol Medical Abortion in Vietnam. Int Fam Plan Perspect 1999, 25(1):10-14.

14. Das V, Jain S, Gupta H, Agarwal A, Agarwal S, Pandey A: Evaluation of newer methods of early pregnancy termination. J Obstet Gynecol India 2005, 55(5):454-456.

15. Honkanen H, Piaggio G, Hertzen H, Bártfai G, Erdenetungalag R, GemzellDanielsson K, Gopalan S, Horga M, Jerve F, Mittal S, et al: WHO multinational study of three misoprostol regimens after mifepristone for early medical abortion. BJOG 2004, 111(7):715-725.

doi:10.1186/1742-4755-8-19

Cite this article as: Woldetsadik et al:: Client preferences and acceptability for medical abortion and MVA as early pregnancy termination method in Northwest Ethiopia. Reproductive Health 2011 $8: 19$

\section{Submit your next manuscript to BioMed Central and take full advantage of:}

- Convenient online submission

- Thorough peer review

- No space constraints or color figure charges

- Immediate publication on acceptance

- Inclusion in PubMed, CAS, Scopus and Google Scholar

- Research which is freely available for redistribution 